\title{
PMT: A Generic Object-Oriented Parallel MultiThread Programming Interface
}

\author{
Xiaoqi Yang \\ Qilong Zheng \\ Guoliang Chen \\ National High Performance Computing National High Performance Computing National High Performance Computing \\ Center at Hefei \\ Center at Hefei \\ Center at Hefei \\ Department of Computer Science and Department of Computer Science and Department of Computer Science and \\ Technology \\ Technology \\ Technology \\ University of Science and Technology University of Science and Technology University of Science and Technology \\ of China \\ of China \\ of China \\ xqyang11@mail.ustc.edu.cn \\ qlzheng@ustc.edu.cn \\ glchen@ustc.edu.cn
}

\begin{abstract}
With the increasing popularity of shared-memory programming model, especially at the advent of Multi-Core processors, more and more programmers hope to write parallel multithread programs conveniently and effectively with the help of parallel multithread programming interface. Unfortunately, these interfaces are not well accepted by sequential programmers, because of incomplete elimination of lower-level details, inflexibility selection of library functions, depending on specific compilers. This paper presents a unique generic object-oriented parallel multithread programming interface, which is designed and implemented to solve the drawbacks of current parallel multithread programming interfaces. Evaluation results show that programmers can benefit a lot from the interface.
\end{abstract}

\section{Categories and Subject Descriptors}

D.1.3 [Programming Techniques]: Concurrent ProgrammingParallel Programming; D.2.13 [Software Engineering]: Generic Object-Oriented Libraries

\section{General Terms}

Algorithms, Performance, Design

\section{Keywords}

Multi-Core, Parallel Multithread Programming

\section{INTRODUCTION}

Compared with sequence programming, parallel programming model is more complex and diversity. Parallel programming models act as an abstraction above hardware and memory architectures, which mainly includes the message-passing model and the shared-memory model [2]. The message-passing model is intuitive and easy to implement on parallel computing environments based on distributed memory, such as MPI and

\footnotetext{
Permission to make digital or hard copies of all or part of this work for personal or classroom use is granted without fee provided that copies are not made or distributed for profit or commercial advantage and that copies bear this notice and the full citation on the first page. To copy otherwise, or republish, to post on servers or to redistribute to lists, requires prior specific permission and/or a fee
}

INFOSCALE 2007, June 6-8, Suzhou, China

Copyright $\odot 2007$ ICST 978-1-59593-757-5

DOI 10.4108/infoscale.2007.200
PVM. However, shared-variable model is concise and intuitive in programming on parallel computers based on shared memory.

As Intel puts two cores on a single piece of silicon and as multisocket systems continue to grow, shared memory systems are going to become the norm. To get the most out of shared memory processing power, programmers must embrace parallel programming. But parallel programming comes with its share of challenges. Data structures are tedious to write, even for experienced developers. Therefore, a good parallel multithread interface is inevitable to generate significant interest among developers, and makes them program conveniently and effectively.

\section{RELATED WORK}

UNIX systems, a standardized C language threads programming interface has been specified by the IEEE POSIX 1003.1c standard. Implementations that adhere to this standard are referred to as POSIX threads, or Pthread. Explicit threading libraries, however, are very complicated to use. QThreads, ZThreads and Boost Threads are advanced object-oriented, cross-platform $\mathrm{C}++$ threading and synchronization libraries. These are system level interfaces without providing support for parallel programming design. OpenMP [3] is a collection of directives and supporting runtime library routines to support multi-threaded application programming. However, it lets the programmer adopt a programming discipline in which a program smoothly evolves from a working sequential program into a working parallel program. In addition, it depends on the specific compiler. Intel Thread Building Block is a $\mathrm{C}++$ runtime-library that supports scalable data parallel programming., while it is still under construction.

\section{The Design and Implementation of PMT}

PMT is the library level solution, and it abstracts and encapsulates the basic functions of Pthread with the help of generic technology [1]. Without depending on any specific compilers, PMT can realize the functions similar to those of OpenMP. In addition, PMT provide more comprehensive parallel computing support, such as support for shared variable, point and thread safe access to complex data structure. Furthermore, PMT realizes the support of abstract data structure due to the drawbacks of OpenMP. For example, the reduction variable can be arbitrary user-defined type, and reduction operators can also extend to any user-defined operators. Based on PMT, the basic structure of parallel 
multithread algorithms can be generated. The parallel multithread realizes the function of one program section, and there are some mechanics to support the synchronization of these threads. Therefore, the correct program result is promised and the program efficiency is improved.

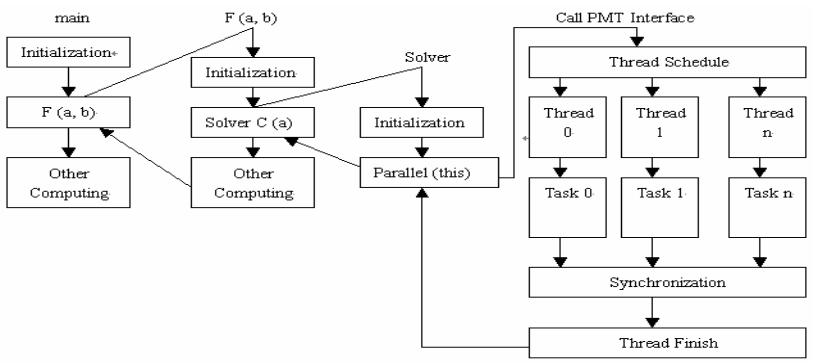

Figure 1. The Calling PMT Model

\section{Thread Management}

PMT provides a more convenient SPMD design interface through simplifying the thread management functions of Pthread. PMT programs begin with an initial thread. When any thread encounters a parallel construct using the parallel keyword then a team of threads is forked.

\section{Basic Parallel Communication Atomics}

PMT encapsulates Pthread's functions to realized mutex, condition variables and semaphores. PMT also provides scoped_lock with the function of exception-safe, which can lock mutex variable automatically at the time of creation and dispose mutex variable at the time of beyond the function scope. In addition, PMT provides the barrier mechanism, which is applied in the field of parallel computing usually.

\section{Parallel Region}

The parallel region of PMT is realized by Macro, so there is no modification or extension of language and no extra compilers required. While compile-directed and specific OpenMP parallel compilers realize the parallel region of OpenMP.

\section{Parallelism Loop}

PMT provides two methods to parallelize loops without any data dependencies. One is the static schedule; the other way is the dynamic schedule. This is done without modifying or extending language and compilers, so programmers only required to modified the sequence loop grammar a little.

\section{Reduction Variable}

The reduction template expression format is presented below: reduction $<\mathrm{T}$, ReductionOperator $>$ local_var (global_var) local_var is the local variable declared in each task, while global_var is applied to store the result generated by the reduction operation.

\section{Shared Variable and Shared Data Structure}

The types of shared variables (such as reduction variable, loop variable and shared pointer) in PMT are not limited to basic types, which can be any type defined by users. That is different of that of OpenMP. PMT defines the concepts of the segment container and the segmented iterator. The difference between segment containers and normal containers is that the data stored segmentary according one specific partition strategy in segmental containers, which can be expressed one sub-data- container or one whole data container for each single parallel task.

Parallel MultiThread Algorithms Structure
The ultimate goal of PMT is to provide programmers with ability of generating parallel generic algorithm.

\section{EVALUATION}

Parallel FFT iteration algorithm [2] is realized by PMT under the evaluation environment (SMP: Four-processor SMP; Processor: Pentium III; Processor Frequency: 866MHz; Memory: $512 \mathrm{MB}$ ). The parallel algorithms realized by PMT flexibility. As can be seen from the figure 2, the program has a good speed ratio. The speed ratio is improved with the number of processors applied.

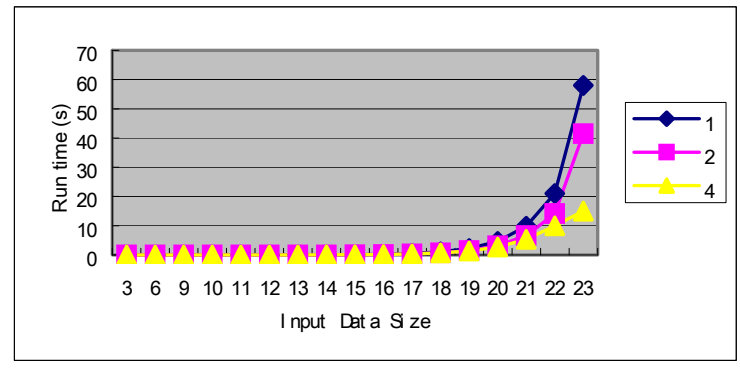

Figure 2. The Evaluation Result

\section{Conclusions and Future Work}

PMT provides the support of parallel task group and common basic communication atomic applied in the parallel algorithm design. It defines rich methods to generate parallel region, and the dynamic and static schedule for parallelizing loop. Furthermore, PMT provides high-level abstraction of shared data mechanism, such as reduction variable, shared variable, shared pointer, variety kinds of shared data containers and so on. Compared with OpenMP, PMT does not depend on any special language extension and compilers. Therefore, it can be applied more widely, and deployed on the shared-memory system more easily, flexibility and expansibility. Currently, we focus our interest on Speculative Parallel Threading [4] technology, which is a promising parallel programming technique for the future multicore architectures and will be incorporated into our PMT system.

\section{ACKNOWLEDGMENTS}

This work was supported by the National Natural Science Fund Key Project (60533020), Intel Higher Research Fund (4507146713), and Anhui Province Natural Science Fund (050420205). Many thanks for Dr. Zhen Yao's previous work related to the project.

\section{REFERENCES}

[1] A. Alexandrescu. Modern $\mathrm{C}++$ Design: Generic Programming and Design Patterns Applied. AW C++ in Depth Series. Addison Wesley, January 2001.

[2] G.Chen. Parallel Computing - Structure, Algorithm and Programming. High Education Press. (1999) 310-318

[3] OpenMP API. http://www.openmp.org/.

[4] Z. Yao, Q .L.Zheng, G.L.Chen and X.Q.Yang, Software Simulation of Speculative Parallel Threading using Transactional Execution, MINI - MICRO SYSTEMS,2007 